\title{
Resiliência Oculta: A Construção Social do Conceito e suas Implicações para Práticas Profissionais junto a Adolescentes em Situação de Risco
}

\author{
Hidden Resilience: The Social Construction of the Concept and \\ its Implications for Professional Practices with At-risk Adolescents \\ Renata Maria Coimbra Libório*,a \& Michael Ungar ${ }^{b}$ \\ ${ }^{a}$ Universidade Estadual Paulista Júlio de Mesquita Filho, Presidente Prudente, Brasil \\ $\&{ }^{b}$ Dalhousie University, Halifax, Canadá
}

\begin{abstract}
Resumo
O presente artigo visa apresentar as principais idéias e conceitos sobre resiliência, tendo como parâmetro a produção de Michael Ungar. Inicialmente será apresentada a perspectiva pós-moderna, na qual discutirse-á sobre a construção social do conceito de resiliência e em seguida será introduzido ao leitor o conceito de "hidden resilience", aqui traduzido como resiliência oculta e relacionado a estratégias de coping nãoconvencionais. As pesquisas com adolescentes no Canadá e em vários países possibilitou a consolidação da proposta elaborada pelo autor, para o qual resiliência refere-se a uma experiência relacionada à capacidade do indivíduo de navegar por recursos associados ao bem-estar e à capacidade de suas famílias e comunidade em prover tais recursos de formas culturalmente significativas. Implicações para práticas profissionais na área da adolescência e juventude serão discutidas.

Palavras-chave: Resiliência Oculta; Adolescentes em Risco; Práticas Profissionais.
\end{abstract}

\begin{abstract}
The purpose of this paper is to present the main concepts and ideas about resilience developed by Michael Ungar. At first, it will be discussed his post-modern approach of resilience and the social construction of the phenomenon. Thereafter, it will be presented the concept of hidden resilience related to unusual coping strategies. Researches carried out with adolescents in Canada and other countries solidified the conception of resilience as an experience associated to the individual's capacity to develop resources associated to his/her well-being and the condition of the individual's family and community to provide such resources in culturally meaningful ways. It will also deal with implications for professional practices with at-risk adolescents.
\end{abstract}

Keywords: Hidden Resilience; At-Risk Adolescents; Professional Practices.

O presente artigo tem como objetivo apresentar e discutir os principais conceitos de resiliência baseado na abordagem de Michael Ungar, em razão da inexistência de publicação em português do material por ele produzido. Consideramos que os estudos em resiliência tem importância fundamental para a área da psicologia, tendo em vista sua capacidade de nos levar a refletir sobre aspectos positivos do desenvolvimento humano, quando indivíduos encontram-se expostos a adversidades signi- ficativas, capazes de colocar em risco a integridade física e psíquica dos mesmos. Atualmente vemos a consolidação da literatura latino americana, especialmente produzida no Brasil, que vem crescendo e acrescentando elementos distintos à literatura da área, tendo em vista as produções anglo-saxãs, tal como as discussões sobre resiliência comunitária (Ojeda, 2005). A produção de Michael Ungar ${ }^{1}$ pode ser bastante útil aos leitores de língua portuguesa e lembramos que muitos de seus quesconhecimentos, debater idéias e discutir sobre pesquisas qualitativas, e a sua brilhante equipe de pesquisadores, Linda Liebenberg, Nora Didkowsky, Cathy Campbell e Amber Raja.

${ }^{1}$ Suas pesquisas têm focado a compreensão de resiliência entre crianças, jovens e famílias através do estudo em várias culturas e contextos. Atualmente vêm conduzindo vários estudos, que podem ser conhecidos através do site www.resilienceresearch.org, incluindo um programa de pesquisa em 14 comunidades em 5 continentes sobre desenvolvimento saudável em jovens em alto-risco. 
tionamentos sobre a literatura de resiliência baseiam-se, em grande parte, da literatura em língua inglesa. No decorrer do artigo pretendemos estabelecer algumas aproximações entre sua abordagem e os enfoques utilizados por autores latino-americanos.

Primeiramente apresentaremos sua compreensão pósmoderna sobre a construção social do fenômeno, de forma a contextualizar a elaboração do conceito de "hidden resilience", possibilitado a partir do trabalho terapêutico desenvolvido por vários anos pelo autor, junto a adolescentes em situação de risco. Durante seus atendimentos, foi possível verificar a presença de estratégias de coping consideradas não-convencionais que foram nomeadas de "hidden resilience" (Ungar, 2001, 2002, 2007) aqui traduzidas por "resiliência oculta".

De acordo com Ungar (2008) nas últimas décadas do estudo da resiliência, pesquisadores têm analisado esse constructo como relacionado ao "desenvolvimento positivo de crianças e adolescentes quando confrontados por adversidades" (p. 218). Um primeiro questionamento feito pelo autor refere-se às abordagens utilizadas na maioria de estudos sobre resiliência conhecidas através da literatura inglesa, especialmente produzida no mundo ocidental.

Embora tais estudos busquem uma contextualização do "risco" e tenham documentado vários processos protetivos que podem prever "resultados positivos" durante o desenvolvimento, tais "resultados" seriam: (a) Baseados em conceitos e valores ocidentais e hegemônicos, enfatizando fatores individuais e relacionais típicos de tais sociedades majoritárias e consequentemente de suas definições sobre funcionamento saudável, que incluem "permanecer na escola, apego com mãe ou outro cuidador, formação de apego seguro com um parceiro durante vida adulta, formas de adaptação não delinquentes" (Ungar, 2008, p. 218). Devido a isso haveria uma arbitrariedade na seleção dos resultados esperados e associados à resiliência, baseados essencialmente em concepções ocidentais (Ungar \& Liebenberg, 2005). (b) Em segundo lugar, na história da produção de maioria do conhecimento em língua inglesa sobre o constructo da resiliência, alguns enfoques não discutiam sobre os aspectos culturais e comunitários que dão sustentação à definição e manifestação de resiliência nas trajetórias de vida dos indivíduos (Ungar, 2004), conduzindo o autor na busca de uma compreensão de resiliência em diferentes culturas e contextos mundiais. A percepção de tal limitação levou Ungar a realizar pesquisas como o "The International Resilience Project" (Ungar, 2006; Ungar \& Liebenberg, 2005) desenvolvido nos anos de 20032005 em 14 comunidades em 5 continentes e o projeto de pesquisa internacional mais recente "Negotiating Resilience: Protective Process of Children in Transition Across Cultures and Contexts" (Ungar, Liebenberg, \& Didkowsky, 2007), que encontra-se em desenvolvimento em 5 países. Dentre as principais questões levantadas nessas pesquisas citamos o seu interesse em compreen- der como diferentes contextos e culturas influenciam na definição de "risco", na compreensão dos processos mediadores associados com resiliência e definições localizadas culturalmente sobre "resultados positivos".

Ungar e demais pesquisadores de sua equipe reforçam a necessidade de entender resiliência como associada à "capacidade do indivíduo em navegar ${ }^{2}$ seu caminho em direção a recursos de bem-estar assim como a capacidade de suas comunidades oferecerem esses recursos de formas culturalmente significativas" (Ungar, Brown, Liebenberg, Cheung, \& Levine, 2008, p. 3). Portanto, resiliência é vista como resultante daquilo que as comunidades definem como funcionamento saudável e socialmente aceito para suas crianças e adolescentes, bem como a capacidade de suas comunidades em prover recursos significativos.

Dentre a preocupação do autor insere-se o questionamento sobre as metodologias de pesquisa usadas para o estudo de resiliência bem como a sua definição, basicamente articulada com "resultados positivos esperados" para o estudo da experiência de populações minoritárias e marginalizadas. Tal preocupação encontra paralelo na literatura produzida no Brasil e na América do Sul, desde o início do século XXI, como podemos ver nas produções dos seguintes autores: Barlach (2005), com suas análises sobre a complexidade da construção do conceito de resiliência, em Cecconello (2003) e De Antoni (2003) a partir da utilização da inserção ecológica como metodologia de estudo junto a famílias em situação de adversidades, no artigo de Libório, Castro e Coelho (2006), no qual os autores analisam as dimensões metodológicas da pesquisa sobre resiliência, em Junqueira e Deslandes (2003) e Pinheiro (2004) que discutem sobre a dimensão ideológica do conceito, a partir do trabalho de Ojeda (2005) com sua perspectiva da resiliência comunitária e sobre as condições coletivas de grupos humanos enfrentarem adversidades, no artigo Yunes e Szymanski (2001) que refletem sobre a relatividade dos conceitos de risco e proteção, e Yunes $(2006,2007)$ que argumenta a favor do discurso crítico sobre resiliência e resiliência familiar.

De acordo com Ungar (2006) a falta de significação cultural do desenho metodológico da pesquisa e a desconsideração de uma interpretação de dados mais articulada com a cultura que está sendo estudada representaria fragilidade nos estudos de resiliência assim como na implicação prática da pesquisa. A tarefa de compreender resiliência, levando-se em consideração culturas e contextos não é uma tarefa simples, pois os estudiosos têm se confrontado com as tensões entre homogeneidade e hetorogeneidade nas formas pelas quais fenômenos relacionados à saúde são conceitualizados. Apesar de tal 
complexidade, Ungar (2008) defende a necessidade de verificarmos o significado de resiliência em diferentes culturas e contextos, visando compreender quais são os recursos disponíveis para sobrevivência em vários contextos que podem se diferir daqueles acessíveis na cultura ocidental.

Essa consideração aos aspectos culturais do constructo de resiliência desafia, de certa forma, aquilo que é aceito como "resultados positivos, saudáveis", bem como os comportamentos normativos. Ungar questiona os sérios limites de uma abordagem mais individualizante de resiliência, que desconsidera os problemas estruturais da sociedade e a ausência de políticas públicas que famílias e comunidades necessitam para se fortalecer e consequentemente nutrir processos de resiliência em suas crianças e adolescentes enfrentando adversidades. Portanto, resiliência, longe de se referir unicamente a traços individuais, associa-se igualmente com as características do lugar social e político ocupado pelas crianças, adolescentes, suas famílias e comunidades. Essa forma menos individualizante de conceber resiliência minimiza a tendência de atribuir ao sujeito de forma singular, a responsabilidade por seu insucesso. De forma bastante enfática Ungar (2005b) afirma que resiliência "não é um estado psicológico interno de bem-estar, nem um conjunto de comportamentos aceitáveis socialmente que ocorrem após a exposição ao risco, nem uma condição que resulta de qualidades inatas tais como, temperamento positivo ou capacidades latentes" (p. 90). Decorrente dessas considerações de Ungar, novamente é possível demarcar aqui a sintonia entre suas concepções e poucos autores de língua inglesa, tais como Martineau (1999) e McCubbin e McCubbin e Froma Walsh, apud Yunes (2007), bem como autores latino-americanos como Junqueira e Deslandes (2003), Klevens, Restrepo e Roca (2000), Libório et al. (2006), Ojeda (2005), Pinheiro (2004), Yunes (2006, 2007) e Yunes e Szymanski (2001).

\section{Um Discurso Construcionista de Resiliência}

A compreensão construcionista de resiliência, como um discurso alternativo sobre o conceito baseia-se no pósmodernismo discutido por Kenneth Gergen (2001), apud Ungar (2004) e fundamenta-se na teoria de Michel Foucault, que explica a "realidade social como construída através da interação e altamente dependente da linguagem que usamos para descrever nossa experiência" (Ungar, 2004, p. 342) e afirma que aqueles que detêm maior poder no controle social do discurso influenciam nas nossas definições de saúde e doença. Tal forma de compreender resiliência nos faz pensar sobre as aproximações de suas idéias e a abordagem ecológica, que tem sustentado há alguns anos grande parte da pesquisa em resiliência. Segundo Ungar, a abordagem ecológica, por ser uma teoria sistêmica, aborda o risco e resiliência através de um enfoque que enfatiza a existência de relações previsíveis entre fatores de risco e proteção, indicando uma causalidade circular e um processo transacional promotor de resiliência. No paradigma ecológico, resiliência tem sido definida basicamente como saúde apesar de adversidades, que pode ser entendida de forma complementar à concepção construcionista por ele defendida, na qual resiliência reflete uma interpretação pósmoderna, definida como "o resultado de negociações entre indivíduos e seus ambientes por recursos que os definem como saudáveis em meio a condições coletivamente vistas como adversas" (Ungar, 2004, p. 342).

O modelo ecológico e hierarquicamente ordenado de fatores de risco e proteção, conforme apresentado no trabalho de Bronfenbrenner nos oferece um modelo muito útil de conceitualização de resiliência, sendo essa dependente das interações dos diferentes níveis do sistema ecológico.

A pesquisa de Ungar nos oferece uma compreensão de resiliência pós-moderna que pode ser entendida como uma ampliação da proposta de Bronfenbrenner e propõe que os indivíduos e discursos sociais nos quais participam, moldam negociações que determinarão se um fator de proteção num nível ou no outro (por exemplo: um relacionamento interpessoal específico, uma interação comunitária ou prática cultural) promoverão resiliência (2008).

Levando em consideração os discursos variáveis sobre funcionamento "saudável" ou crescimento psicológico dentro de culturas e contextos específicos, Ungar mostra que a interferência de cada fator de proteção, em distintos níveis ecológicos irão influenciar resultados desenvolvimentais diferenciados, dependendo da construção social de cada fator e o significado que lhe é atribuído. Pensemos no exemplo de uma família, criando sua filha em um contexto no qual a educação escolar não é algo valorizado, ou se considerássemos sociedades pré-industriais nas quais a escolarização poderia não ser um caminho para a formação de papéis sociais adultos bem sucedidos, um sistema educacional disponível pode não ser percebido como um fator de proteção, ou seja, que não contribua para os processos de resiliência.

O modelo de Bronfenbrenner, entretanto, não é contestado por Ungar, que introduz um relativismo pós-moderno às discussões sobre quais fatores com quais combinações (que podem ser múltiplas) afetam a resiliência.

Estamos entendendo, portanto, que o trabalho de Bronfenbrenner implica em categorias fixas, enquanto o trabalho de Ungar mostra que os processos de navegação das crianças e adolescentes em direção a recursos protetivos dependerá da qualidade dos recursos que têm maior relevância para eles, bem como da capacidade de negociação com aqueles que fornecem os recursos aos quais as crianças e adolescentes percebem que são necessários para seu bem-estar. Retomamos como exemplo, contextos sociais e culturais nos quais o processo de escolarização não é significativo para crianças e adolescentes. Nessa situação, a inserção mais precoce em atividades de aprendizado associados com os negócios de 
sua família ou a participação em ritos de passagem apropriados a sua idade, que marcam a transição para a vida adulta, poderão ser mais reconhecidos como caminhos em direção à resiliência do que a freqüência escolar. Visto por esse prisma, um recurso como a escola não é facilmente compreendido sem a apreciação dos múltiplos aspectos de resiliência: os sistemas individuais e coletivos de atribuição de significados, a cultura e o acesso ao recurso (há disponibilidade, por exemplo, de escola, caso a criança e o adolescente desejem frequientá-la?).

Retomando, a noção de resiliência enquanto uma construção social sugere uma relação menos definida e menos causal entre níveis ordenados de sistemas ecológicos. Ao invés disso, um modelo menos hierárquico é necessário para apreender negociações mais caóticas em direção a fatores relacionados à resiliência, quando crianças, adolescentes, suas famílias, instituições e comunidades interagem com lugares discursivos que estão constantemente em fluxo.

Portanto, pesquisas que se embasam na concepção construcionista de resiliência, têm observado que as relações entre fatores de risco e proteção são não-sistêmicas e não-hierárquicas, de tal forma que na descrição das relações entre tais fatores, quando se toma como parâmetro culturas e contextos sociais e políticos distintos, essas apresentam-se como "caóticas, complexas, relativas e contextuais" (Ungar, 2004, p. 342).

Com relação aos fatores de resiliência, na perspectiva construcionista, eles são multidimensionais, específicos a cada contexto e podem prever "resultados associados à saúde, de acordo com as definições apresentadas pelos indivíduos e seu grupo de referência social" (Ungar, 2004, p. 344), compensando riscos que são também definidos pelo grupo cultural. Para proteger o indivíduo contra as ameaças ao seu bem estar, devemos explorar a disponibilidade de recursos oferecidos em determinada cultura (que podem variar de um grupo cultural a outro).

No que se refere à definição de saúde, na perspectiva construcionista, essa é construída, abrangendo uma pluralidade de comportamentos e significados, de tal forma que a descrição dos fenômenos associados à resiliência aprofundam nossa compreensão sobre as maneiras pelas quais "populações em situação de altorisco descobrem e nutrem resiliência de formas muitas vezes invisíveis aos olhos dos profissionais de saúde" (Martineau, 1999; Ungar, 2001).

Ungar (2004) frisa, entretanto, que não devemos ver a perspectiva construcionista como sendo a mais correta sobre resiliência, assim como ela não deve ser vista como a única capaz de interpretar os fenômenos relacionados a bem-estar. Sua concepção de resiliência convive com a diversidade de formas de entender a forma como o fenômeno é nutrido e mantido em diversos contextos sociais e culturais, assim como com a pluralidade de significados que os indivíduos podem associar a sua própria auto-construção como "resiliente" ou "não resiliente". Tais idéias deram sustentação à construção do conceito de resiliência oculta, que será apresentada em tópico a seguir.

Ao apresentar a visão construcionista, a intenção de Ungar é ajudar a questionar as relações de poder existentes no processo de atribuição de significados aos conceitos de saúde, que são oferecidas como "verdades", mas são pouco relevantes e sem sentido para alguns grupos sociais e culturais distintos e compreender que resiliência está mais associada aos discursos que são construídos e específicos a cada contexto, do que a medidas objetivas de saúde, que tem um caráter mais universalizado.

A perspectiva construcionista esclarece que parâmetros objetivos de análise de resiliência, fatores ou mecanismos de risco e proteção não deveriam ser enfocados como tendo caráter universal, em razão da dinamicidade, relatividade e não-causalidade linear entre tais fenômenos (Martineau, 1999; Ungar, 2004; Yunes \& Szymansky, 2001), de forma que inexiste um conjunto de condições que podem ser vistos como protetores para todas as crianças e adolescentes em âmbito global. A consideração acerca da ambiguidade em relação ao conceito de risco, complexificando consequentemente a definição de resiliência, sua especificidade contextual, assim como da concepção de saúde e bem-estar deve ser reconhecida se pretendemos compreender de forma mais significativa a resiliência enquanto um fenômeno contextual e cultural, sendo essencial se queremos estudá-la, portanto, entre culturas.

Assumindo essa perspectiva podemos enfrentar a limitação apontada por Kaplan (1999), citado por Ungar (2004, p. 351), referente à definição de resiliência, que é atada a "julgamentos normativos relativos a resultados particulares". Além da necessidade de contextualizar a compreensão de resiliência, Kaplan (1999), nos lembra da dimensão subjetiva, dos sujeitos singulares, a respeito do que são resultados positivos ou negativos; ou seja, mesmo dentro de um grupo cultural específico, que definem certos resultados como desejáveis socialmente, um determinado sujeito pode interpretar subjetivamente tal resultado como indesejável, de tal forma que "do ponto de vista subjetivo, o indivíduo pode estar manifestando resiliência, enquanto do ponto de vista social o indivíduo pode estar manifestando vulnerabilidade" (Kaplan, 1999, citado em Ungar, 2004, p. 351). Portanto, aliada a dimensão contextual e cultural, aspectos subjetivos também devem ser considerados, tal como é lembrado por Boyden e Mann (2005), Martinez (2001) e Woodhead (2004), segundo os quais os efeitos da adversidade sobre a criança ou adolescente "são determinados não somente pela natureza objetiva do ato ou situação, mas também pela experiência subjetiva da criança ou adolescente sobre a situação" (p. 21). A constatação da influência da cultura, contexto e subjetividade na construção dos conceitos de resiliência, risco e saúde nos remete à necessidade dos pesquisadores, durante o desenvolvimento de pesquisas qualitativas e/ou quantitativas, se aproximarem das construções coletivas e individuais 
do conceito de resiliência entre os grupos culturais que estarão participando do estudo, que pode evitar uma postura etnocêntrica (Ungar, 2005a).

A concepção construcionista de resiliência nos remete ainda a pertinência do uso desse construto em algumas culturas, visto que sua elaboração foi feita no contexto de estudos com populações ocidentais, através da participação de profissionais da saúde inseridos nessa cultura particular (Boyden \& Mann, 2005). A preocupação com esse aspecto foi levada em consideração por Ungar (2001, 2005a, 2008) que em suas pesquisas busca a compreensão dos profissionais e dos próprios participantes das pesquisas sobre o que eles entendem por "resiliência", apresentada a eles como algo associado a crescer bem, mesmo estando sob stress / dificuldades (Ungar, Liebenberg, et al., 2007), investigando e explorando os significados desses conceitos junto ao grupo cultural participante da pesquisa, que podem expressar suas crenças e valores sobre o que entendem por esse "crescer bem", mesmo desconhecendo o termo "resiliência". Enfatizamos que alguns autores brasileiros também partilham essa preocupação, conforme pode ser visto nos trabalhos de Cecconello (2003), De Antoni (2003), Junqueira e Deslandes (2003), Pinheiro (2004), Yunes (2007) e Yunes e Szymanski (2001).

Segundo Ungar (2004), baseado em resultados de estudos qualitativos e evidências práticas obtidas através de narrativas de vida, sugere que resiliência pode ser melhor compreendida fenomenologicamente, ao invés de ser concebida como um fato objetivo, sendo, portanto, compreendida como "negociação bem sucedida dos indivíduos em busca de recursos de saúde, cuja definição de sucesso baseia-se na experiência de reciprocidade entre eles e as construções sociais de bem-estar que moldam a interpretação de seu status de saúde" (p. 352).

Considerando sua definição de resiliência, encontramos adolescentes e jovens que, mesmo tendo sido rotulados de delinquentes e perigosos, mantém, de forma surpreendente, um bom crescimento psicológico (Ungar, 2001, 2002), conforme pode ser visto nas pesquisas de Cirillo (2000), Morgan (1998) e Ungar e Teram (2000), apud Ungar (2004). Tais pesquisas desafiam a noção homogeinizadora de "comportamento saudável", bem como o esteriótipo daquilo que consideramos "resiliente", na medida em que comportamentos problemáticos e negativos podem ser, na verdade, sinais de saúde em alguns contextos, pois através dos mesmos adolescentes e jovens podem acessar recursos viabilizadores de saúde (tais como sentimento de pertencimento à comunidade, vínculos significativos). Pesquisas com populações em situação de rua no Brasil também evidenciam essa perspectiva (Botelho, Silva, Kassab, \& Leite, 2008; Paludo \& Koller, 2005).

Ressaltamos, entretanto, que esse discurso construcionista de risco e resiliência não necessariamente define todos os comportamentos rotulados de desviantes como funcionalmente adaptativos, ou seja, tal compreensão deve envolver somente aqueles comportamentos problemáticos que ajudam os indivíduos a experienciar a resiliência. No próximo tópico exploraremos melhor essa concepção.

\section{A Resiliência Oculta}

A elaboração do conceito de resiliência oculta tem suas raízes na tese de doutoramento de Ungar (1995), cujo objetivo era estudar a relação entre a experiência de empoderamento e saúde mental entre adolescentes que haviam passado por atendimentos nas áreas da saúde mental, sistema de proteção a infância e sistema judiciário. Dentre os resultados de sua pesquisa, de forma inesperada, Ungar (2001) constatou a presença da resiliência enquanto uma construção social dos adolescentes em alto risco e de seus cuidadores tanto dentro como fora das instituições de atendimento. Tal constatação associava-se com o conjunto de conhecimentos elaborados por alguns pequisadores (Gregson, 1994; Ungar \& Teram, 2000; Weis \& Fine, 1993), citados por Ungar (2001), que vinham criticando os viéses adultocêntricos presentes em estudos com adolescentes sobre as formas de experienciar saúde e doença mental, que não conseguiam enxergar "aspectos promotores de saúde por trás de comportamentos problemáticos entre jovens em alto-risco" (Ungar, 2001, p. 137). Tais comportamentos seriam estratégias que adolescentes vivendo em contextos pobres em recursos usariam para sustentar um senso de si próprios como saudáveis e "resilientes", o que nos desafia a pensar sobre as diferentes formas de coping acionadas por tais jovens. Ungar (2001) partilha da afirmação feita por pesquisadores da área da saúde mental que afirmam

adolescentes rotulados de problemáticos aumentam seu status de saúde através de empoderamento pessoal e social associado a comportamentos tais como uso de álcool e drogas, atividade sexual precoce, morar na rua, uso de armas, vadiagem, envolvimento em comportamentos criminosos, e até tentativas de suicídio (p. 138).

Por mais paradoxal que tal afirmação pareça, os autores querem expressar sua preocupação sobre o quanto nós, enquanto profissionais da saúde e áreas afins, ignoramos estratégias de coping que fogem do que é estereotipado como sendo associado a bem estar, e dessa forma impactamos negativamente no desenvolvimento de resiliência desses adolescentes, ao colaborarmos com a construção de identidades saturadas de problema, ignorando ainda a possibilidade deles estarem fazendo escolhas racionais, frente a uma realidade com tão pouco acesso a recursos mais "satisfatórios" para alcançar autoestima, bem estar, satisfação, sensação de poder e controle sobre suas vidas. Estudos recentes sobre crianças e adolescentes em situação de rua (Botelho et al., 2008; Invernizzi, 2003; McAdam-Crisp, Aptekar, \& Kironyo, 2005; Paludo \& Koller, 2005) envolvidos em conflito 
armado (Betancourt, 2008; Boothby, 2006; Cortes \& Buchanan, 2007; Denov \& Maclure, 2007), exploração sexual (Montgomery, 2007) e outras formas de trabalho infantil (Libório \& Pessoa, 2008; Liebel, 2004; Woodhead, 2004), têm apresentado resultados de suas pesquisas que vão ao encontro ao conceito de resiliência oculta elaborado por Ungar. O reconhecimento dessa dimensão expressa através de comportamentos "desviantes" não significa que nós, assim como os autores acima citados, defendemos esses comportamentos como sempre sendo benéficos para toda e qualquer criança e adolescente em qualquer contexto social e cultural, e que essas formas de acesso a bem estar e relacionamentos significativos tragam somente aspectos construtivos, como se assumíssemos uma visão muito ingênua sobre o tema, ou ignorando a estrutura social e política de cada comunidade onde tais comportamentos se materializam.

Considerando que resiliência, como um resultado, deriva (pelo menos em parte) das "oportunidades que crianças e adolescentes têm de acessar apoios psicológicos, emocionais, relacionais e instrumentais que precisam para crescer bem, enquanto vivendo sob circunstâncias adversas" (Ungar, 2007, p. 2) perguntamos: o que fazem crianças e adolescentes quando eles não têm acesso a recursos, que poderiam ser oferecidos por profissionais e seus serviços, que auxiliariam na construção de bem-estar, obtenção de status, reconhecimento social? Como eles sobrevivem e vivem bem? Quando caminhos mais "saudáveis" associados ao bem-estar estão bloqueados ou ausentes, que formas de coping são possíveis e viáveis para tais crianças e adolescentes? Tais questões certamente nos apontam para a responsabilidade da sociedade e do Estado no oferecimento de recursos "mais saudáveis" favorecedores de resiliência.

O reconhecimento dessa situação complexa, levou Ungar a ampliar o conceito de resiliência, criando o termo hidden resilience, resiliência oculta, que se relaciona a comportamentos específicos associados com perigo (dangerous), afastado dos padrões (deviant), delinquência e desordem (em ingles, os 4 Ds), que funcionam como caminhos não padronizados de acesso à saúde, que podem se apresentar nas vidas de adolescentes e jovens marginalizados de alto-risco (Ungar, 2001). Portanto, resiliência oculta está se manifestando quando se utiliza formas não-convencionais (que podem vincular-se a: associação a gangs, uso de drogas, trabalho infantil, agressividade, bullying, abandono escolar) de fortalecimento de identidade e acesso a experiência de resiliência (através de: aumento de competências, aquisição de auto-estima, pertencimento a um grupo, estabelecimento de relações interpessoais significativas, ter poder de decisão). Disso decorre que os comportamentos não-convencionais não representam somente sinais de vulnerabilidade, ao invés disso, alguns comportamentos problemáticos podem ser manifestações culturalmente e contexualmente significativas de resiliência, uma forma oculta que é negligenciada por profissionais que não compartiham o mesmo contexto do jovem e de sua comunidade (Ungar, 2007). O conceito de resiliência oculta nos ensina que os caminhos de acesso a esse fenômeno podem ser plurais e inesperados, pois podemos encontrar histórias de sobrevivência que não seriam celebradas quando vistas pelo olhar de um observador externo. Ungar explica que as crianças e adolescentes que crescem bem frente adversidades, "fazem isso com qualquer coisa que estiver disponível e acessível, percebidos como úteis para sua manutenção" (2007, p. 3), ela tenta obter o melhor, dentre as poucas opções que estão ao seu redor, ou seja, os recursos disponíveis e acessíveis circunscrevem a experiência de resiliência. Por vezes, os comportamentos não-convencionais podem representar uma "escolha" imprópria (do ponto de vista dos profissionais) de adolescentes que em seus contextos, não tem nenhuma outra escolha disponível, sendo a única solução apresentável, quando outros recursos promotores de saúde são tão escassos.

Para compreender tais afirmações, vamos pensar no exemplo de um adolescente que não frequenta a escola, mas isso não impede o desenvolvimento da auto-valorização, independência, relacionamentos significativos, reconhecimento da comunidade ou auto-eficácia, que podem ser obtidos através de sua contribuição como membro de um grupo de moradores de rua, de gangs ou através de envolvimento no trabalho (Botelho et al., 2008; Invernizzi, 2003; Libório \& Pessoa, 2008; Liebel, 2004; Ungar, 2007; Woodhead, 2004). Se pretendemos oferecer para o tal adolescente formas mais "convencionais" de acesso à resiliência, a substituição deverá ser feita através de oferecimento de soluções realistas, acessíveis e significativas para ele e sua comunidade, e que tais substituições sejam capazes de fazer o jovem experienciar aquilo que ele adquiria através das formas "não-convencionais". Portanto, se o adolescente obtem benefícios através do trabalho precoce ou envolvimento em gang, tais benefícios deverão continuar sendo obtidos, através dos recursos mais "convencionais" a serem oferecidos a ele. Essa concepção tem implicações em âmbito das intervenções, tais como na resistência ao atendimento e na condução de uma prática profissional que leve em conta a pluralidade de formas de acesso a resiliência e a lógica daqueles vivendo sob adversidades. A questão da intervenção é abordada por Ungar em vários artigos (2001, 2006, 2005a, 2005b, 2007, 2008).

\section{Definindo Resiliência}

Apresentaremos as formulações mais recentes de Ungar derivadas de sua abordagem construcionista de resiliência e das pesquisas envolvendo adolescentes de vários países, contando com a participação de pesquisadores em âmbito internacional, que podem ser encontradas em vários artigos e livro (Ungar, 2005a, 2006, 2008; Ungar et al., 2008; Ungar, Brown, et al., 2007; Ungar, Lee, Callaghan, \& Boothroyd, 2005; Ungar \& 
Liebenberg, 2005) ${ }^{3}$. A pesquisa International Resilience Project (IRP) realizada em 14 comunidades nos 5 continentes examinou padrões de coping e processos protetivos nas vidas de aproximadamente 1500 adolescentes expostos a riscos, avaliados de acordo com os consultores da pesquisa dos diferentes países, que apresentavam distintas realidades culturais (Ungar, 2006; Ungar et al., 2005; Ungar \& Liebenberg, 2005). Os riscos abrangiam: pobreza, guerra, deslocamento social, desintegração cultural, genocídios, violência, marginalização, abuso de álcool e drogas, quebra dos vínculos familiares, doenças mentais nas crianças e/ou pais e gravidez precoce (Ungar, Brown, et al., 2007). A obtenção de dados qualitativos junto a 89 jovens (12-23 anos) do sexo masculino e feminino de regiões do mundo, apresentando grande e significativa variabilidade geográfica e cultural, tais como Canadá, Africa do Sul, Colômbia, Palestina, China, Israel, Estados Unidos, Gambia e Rússia, que estavam expostos a no mínimo 3 situações de riscos significativos de acordo com sua cultura, possibilitou a construção de uma imagem acerca de alguns aspectos comuns, mas principalmente da heterogeneidade dos contextos culturais nos quais os adolescentes buscavam os recursos para sustentar seu próprio bem-estar.

A partir da pesquisa do IRP, foram identificadas constelações de processos protetivos encaminhando os adolescentes para um crescimento psicológico. Os pesquisadores verificaram a presença de 7 temas relevantes em comum (acesso a recursos materiais; experiências de justiça social, acesso a relacionamentos interpessoais que oferecem apoio; identidade pessoal desejável; senso de coesão; experiências de poder e controle pessoal; aderência cultural) que compareceram nas narrativas dos 89 adolescentes participantes que foram denominados de "tensões" pelos pesquisadores, que ao serem resolvidas (de formas diversas) auxiliavam o adolescente em seu caminho para um crescimento psicossocial "positivo", associado com bem-estar e resiliência, de acordo com critérios de suas culturas e contextos. As tensões relacionam-se aos processos protetivos, sendo que os participantes da pesquisa ilustraram com suas histórias o aparecimento dessas tensões, em maior ou menor número, e formas de solucioná-las de acordo com valores culturais e recursos disponíveis em cada realidade cultural, social e econômica. A resolução das tensões é um processo dinâmico, cuja relação entre variáveis é não-linear e não-casual e pode encaminhar os adolescentes em direção a processos de resiliência, por caminhos convencionais ou não-convencionais (associados à resiliência oculta).

Dentre os aspectos mais importantes discutidos por Ungar, Brown et al. (2007), referem-se à: não há evidên-

\footnotetext{
${ }^{3}$ Sugerimos que aqueles leitores interessados em compreender mais detalhadamente os aspectos metodológicos da pesquisa, tais como critérios para a seleção dos participantes, instrumentos utilizados e formas de análise dos dados coletados, possam acessar as referências mencionadas nesse artigo.
}

cias que uma forma de resolver as sete tensões é melhor do que outra, indicando que cada jovem particularmente encontrava formas únicas de obter sucesso na superação das tensões. Ou seja, não haveria critério objetivo para avaliar "resultados positivos", pois tal avaliação é influenciada por aspectos pessoais, sendo o sujeito aquele que avaliará se sua vida pode ser entendida como sendo ou não "bem-sucedida", num determinado período. As sete tensões são dinâmicas, interagem entre si e podem se expressar de diferentes formas através do tempo:

Acesso a Recurso Material. Se refere à possibilidade do adolescente acessar estruturas sociais que garantam assistência financeira e a concretização de necessidades básicas (alimento, abrigo, roupas, acesso a cuidados médicos e educação de qualidade e emprego).

Experiências de Justiça Social. Ao expandir seus relacionamentos, os adolescentes desenvolvem a capacidade de, individualmente e coletivamente, reivindicar seus direitos. Vivências de preconceito e de não acesso aos privilégios político sociais funcionam como catalizadores de conscientização, resistência, solidariedade, crença em um poder espiritual e enfrentamento da opressão.

Acesso a Relacionamentos Interpessoais que Oferecem Apoio. Os relacionamentos significativos incluem redes compostas por membros familiares, grupo de pares, demais adultos da comunidade, professores, conselheiros, modelos de identificação e amigos íntimos (associados a suporte emocional, experiências de confiança, amor, cuidado e compaixão).

Desenvolvimento de uma Identidade Pessoal Fortalecida. Um senso de individualidade é negociado através das relações com os outros. O processo de formação de identidade é uma co-construção através de interações em espaços discursivos mútuos.

Experiências de Senso de Coesão com Outros. Em contraste com o tópico da individualidade, há a necessidade de estabelecer uma relação balanceada entre o senso pessoal de responsabilidade com o dever e compromisso com a comunidade; tal conceito foi emprestado da teoria do Capital Social de Bourdieu (Ungar, Brown, et al., 2007).

Experiências de Poder e Controle Pessoal. Essa tensão relaciona-se a "auto-confiança do adolescente e sua capacidade em tomar conta de si próprio e sua convicção sobre a capacidade de promover mudanças que assegurem recursos materiais e relacionamentos" (Ungar et al., 2008, p. 8).

Aderência Cultural. Refere-se à capacidade de aderir (ou ficar em oposição) às normas culturais, crenças e valores de sua comunidade, o que implica em negociações complexas com os cuidadores e comunidades, explicitando os conflitos entre culturas globais e locais.

A definição de resiliência construída a partir dessa pesquisa (IRP) nos instiga a questionar conceitos de resiliência entendidos como um conjunto de processos desenvolvimentais e resultados positivos universais previsíveis e regulares, expressos por adolescentes enfren- 
tando adversidades. Enquanto um adolescente, por exemplo, pode buscar o fortalecimento de identidade, relacionamentos significativos e experiência de poder através do ambiente escolar, outro adolescente, para quem a escola não ofereceu tais alternativas promotoras de resiliência, pode buscar através do envolvimento com gangs, o acesso a tais recursos, nos mostrando dois caminhos distintos de resolução das tensões, em busca de resultados associados à resiliência e bem-estar, sendo um deles mais convencional (escola-rização) e outro nãoconvencional (associação com gangs) vinculado ao conceito de resiliência oculta.

Para finalizar a apresentação teórica, definimos abaixo o conceito de resiliência assumida por Ungar em seus estudos mais recentes:

Primeiramente é a capacidade dos indivíduos navegarem por recursos que mantém bem-estar; em segundo lugar é a capacidade dos ambientes físicos e sociais oferecerem tais recursos e em terceiro lugar, é a capacidade dos indivíduos, suas famílias e comunidades negociarem recursos culturalmente significativos a serem partilhados. É esse processo duplo de navegação através de recursos disponíveis, bem como a negociação por recursos a serem proporcionados de forma valorizada pelos adolescentes, envolvendo tanto o indivíduo e seus ambientes em um processo dinâmico, conduzindo a bem estar. (Ungar et al., 2008, p. 2).

Resiliência deve ser compreendida como um estado dinâmico de tensão entre indivíduos, famílias, comunidades e culturas, não sendo um estado permanente do ser.

\section{Algumas Implicações}

Ao considerarmos a realidade brasileira é importante refletirmos sobre as condições estruturais que apóiam o desenvolvimento "saudável" de crianças e adolescentes aos quais faltam recursos individuais, familiares, comunitários e sócio políticas que sustentam saúde, quando múltiplas adversidades são enfrentadas. Tal análise deverá verificar como os sistemas de atendimento são oferecidos, desenhados, administrados e integrados, pois seu funcionamento influencia o cotidiano das crianças e adolescentes usuários dos serviços, de forma a dar apoio no percurso de resiliência, como podem também intensificar os riscos já vividos (ao patologizarem e rotularem os usuários e não dar atenção às suas reais necessidades).

Portanto, ao elaborarmos políticas públicas e projetos de intervenção na área da infância e adolescência em situação de risco, devemos prestar atenção na estruturação dos serviços, de forma a poder atender as necessidades e desejos que as crianças e /ou adolescentes suprem quando inseridos nas condições consideradas adversas pelos gestores e profissionais, pois se torna inócua uma intervenção que não considere o "lugar" ocupado pela situação de risco identificada (tais como: situação de rua, trabalho infantil, gangs) na vida do sujeito. Precisamos avaliar quais recursos devemos oportunizar às crianças e adolescentes focos de nossa intervenção, na medida em que os mesmos só serão significativos e favorecedores de resiliência caso a eles forem atribuídos as mesmas funções exercidas pela situação de risco vivida.

As intervenções deveriam ser desenhadas de forma contextualizada e se dirigirem às sete tensões simultaneamente, valorizando a aderência cultural, enfrentando os preconceitos e demais injustiças sociais vivenciados, que favoreçam a expressão de poder e controle pessoal (autodeterminação), oferecendo oportunidades de estabelecimento de relacionamentos significativos e construção de identidades fortalecidas.

Outra implicação decorrente refere-se às formas pelas quais profissionais que atuam em projetos sociais e instituições escolares em nossa realidade enfrentam comportamentos não aceitos socialmente, ou seja, não convencionais (por exemplo: bullying, envolvimento com tráfico de drogas, gravidez precoce), apresentados pelas crianças e adolescentes com os quais trabalham. Ungar nos convida a olhar com maior rigor e proximidade os significados que podem estar invisíveis, quando analisados pelo prisma dos usuários de nossos serviços, nos dando indícios de uma forma peculiar de resiliência, a resiliência oculta, que também salienta caminhos possíveis de fortalecimento pessoal e bem estar.

\section{Referências}

Barlach, L. (2005). O que é resiliência humana? Uma contribuição para a construção do conceito. Dissertação de Mestrado não-publicada, Instituto de Psicologia, Universidade de São Paulo, SP.

Betancourt, T. S. (2008). Child soldiers: Reintegration, pathways to recovery and reflections from the field. Journal of Developmental \& Behavioral Pediatrics, 29(2), 138-141.

Boothby, N. (2006). What happens when child soldiers grow up: The Mozambique case study. Intervention, 4(3), 244259.

Botelho, A. P., Silva, S. L. F., Kassab, M. J., \& Leite, L. C. (2008). Meninos de rua: Desafiliados em busca de saúde mental. Psicologia em Estudo, 13(2), 361-370.

Boyden, J., \& Mann, G. (2005). Children“s risk, resilience and coping in extreme situations. In M. Ungar (Ed.), Handbook for working with children and youth: Pathways to resilience across cultures and contexts (pp. 3-25). Thousand Oaks, CA: Sage.

Cecconello, A. M. (2003). Resiliência e vulnerabilidade em famílias em situação de risco. Tese de Doutorado nãopublicada, Instituto de Psicologia, Universidade Federal do Rio Grande do Sul, Porto Alegre, RS.

Cortes, L., \& Buchanan, M. J. (2007). The experience of Columbian child soldiers from a resilience perspective. International Journal for the Advancement of Counselling, 29(1), 43-55.

De Antoni, C. (2003). Coesão e hierarquia em famílias com história de abuso físico. Tese de Doutorado não-publicada, Instituto de Psicologia, Universidade Federal do Rio Grande do Sul, Porto Alegre, RS. 
Denov, M., \& Maclure, R. (2007). Turnings and epiphanies: Militarization, life histories and the making and unmaking of two child soldiers in Sierra Leone. Journal of Youth Studies, 10(2), 243-261.

Invernizzi, A. (2003). Street working children and adolescents in Lima: Work as an agent of socialization. Childhood, 10(3), 319-341.

Junqueira, M. F. P. S., \& Deslandes, S. F. (2003). Resiliência e maus-tratos à criança. Cadernos de Saúde Pública, 19(1), 227-235.

Klevens, J., Restrepo, O., \& Roca, J. (2000). Some factors for explaining resilience among young men in Colômbia. Revista de Salud Publica, 2, 165-172.

Libório, R. M. C., Castro, B. M., \& Coelho, A. E. L. (2006). Desafios metodológicos para a pesquisa em resiliência: Conceitos e reflexões críticas. In D. D. Dell 'Aglio, S. H. Koller, \& M. A. M. Yunes (Eds.), Resiliência e Psicologia Positiva: Interfaces do risco a proteção (pp. 89-117). São Paulo, SP: Casa do Psicólogo.

Libório, R. M. C., \& Pessoa, A. S. G. (2008, jun.). Crianças, adolescentes e trabalho: Caracterização e atribuição de significado. Paper presented at the III Simpósio Internacional sobre Juventude Brasileira no Mundo Contemporâneo: Desafios e Perspectivas, Goiânia, GO.

Liebel, M. (2004). A will of their own: Cross cultural perspectives on working children. New York: Zed Books.

Martineau, S. (1999). Rewriting resilience: A critical discourse analysis of childhood resilience and the politics of teaching resilience to "kids at risk". Unpublished doctoral dissertation, University of British Columbia, Vancouver, Canada.

Martinez, A. M. (2001). Trabajo infantil y subjetividad: una perspectiva necesaria. Estudos de Psicologia (Natal), 6(1), 235-244.

McAdam-Crisp, J., Aptekar, L., \& Kironyo, W. (2005). The theory of resilience and its application to street children in the minority and majority worlds. In M. Ungar (Ed.), Handbook for working with children and youth: Pathways to resilience across cultures and contexts (pp. 71-88). Thousand Oaks, CA: Sage.

Montgomery, H. (2007). Working with child prostitutes in Thailand: Problems of practice and interpretation. Childhood, 14(4), 415-430.

Ojeda, E. N. S. (2005). Uma concepção latino-americana: A resiliência comunitária. In A. Melillo \& E. N. S. Ojeda (Eds.), Resiliência: Descobrindo as próprias fortalezas (pp. 4757). Porto Alegre, RS: Artmed.

Paludo, S. S., \& Koller, S. H. (2005). Resiliência na rua: Um estudo de caso. Psicologia: Teoria e Pesquisa, 21(2), $187-$ 195.

Pinheiro, D. P. N. (2004). A resiliência em discussão. Psicologia em Estudo, 9(1), 67-75.

Ungar, M. (1995). A naturalistic study of the relationship between the process of empowerment and mental health during adolescence. Unpublished doctoral dissertation, Wilfrid Laurier University, Waterloo, Canada.

Ungar, M. (2001). The social construction of resilience among "problem" youth in out-of-home placement: A study of healthenhancing deviance. Child and Youth Care Forum, 30(3), 137-154.

Ungar, M. (2002). Playing at being bad: The hidden resilience of troubled teens. Nova Scotia, Canada: Pottersfied Press.

Ungar, M. (2004). A constructionist discourse on resilience: Multiple contexts, multiple realities among at-risk children and youth. Youth and Society, 35(3), 341-365.
Ungar, M. (2005a). Introduction: Resilience across cultures and contexts. In M. Ungar (Ed.), Handbook for working with children and youth: Pathways to resilience across cultures and contexts (pp. xv-xxxix). Thousand Oaks, CA: Sage.

Ungar, M. (2005b). A thicker description of resilience. The International Journal of Narrative Therapy and Community Work, 3/4, 89-95.

Ungar, M. (2006). Nurturing hidden resilience in at-risk youth in different cultures. Journal of the Canadian Academy of Children and Adolescence Psychiatry, 15(2), 53-58.

Ungar, M. (2007). Contextual and cultural aspects of resilience in child welfare settings. In I. Brown, F. Chaze, D. Fuchs, J. Lafrance, S. McKay, \& S. T. Prokp (Eds.), Putting a human face on child welfare: Voices from the Prairies (pp. 1-23). Regina, Canada: Prairie Child Welfare Consortium.

Ungar, M. (2008). Resilience across cultures. British Journal of Social Work, 38, 218-235.

Ungar, M., Brown, M., Liebenberg, L., Cheung, M., \& Levine, K. (2008). Distinguishing differences in pathways to resilience among Canadian youth. Canadian Journal of Community Mental Health, 27(1), 1-13.

Ungar, M., Brown, M., Liebenberg, L., Othman, R., Kwong, W. M., Armstrong, M., et al. (2007). Unique pathways to resilience across cultures. Adolescence, 42(166), 287-310.

Ungar, M., Lee, A. W., Callaghan, T., \& Boothroyd, R. A(2005). An international collaboration to study resilience in adolescents across cultures. Journal of Social Work Research and Evaluation, 6(1), 5-21.

Ungar, M., \& Liebenberg, L. (2005). The international resilience project: A mixed methods approach to the study of resilience across cultures. In M. Ungar (Ed.), Handbook for working with children and youth: Pathways to resilience across cultures and contexts (pp. 211-226). Thousand Oaks, CA: Sage.

Ungar, M., Liebenberg, L., \& Didkowsky, N. (2007). Negotiating resilience: Protective process of children in transitiono across cultures and contexts - Research manual. Unpublished manuscript, School of Social Work, Dalhousie University, Halifax, Canada.

Yunes, M. A. M. (2006). Psicologia Positiva e resiliência: Foco no indivíduo e na família. In D. D. Dell‘Aglio, S. H. Koller, \& M. A. M. Yunes (Eds.), Resiliência e Psicologia Positiva: Interfaces do risco a proteção (pp. 45-68). São Paulo, SP: Casa do Psicólogo.

Yunes, M. A. M. (2007). The ideological trap of the advocacy's discourse on resilience in poor families. Journal of Applied Psychology, 3(1), 26-33.

Yunes, M. A. M., \& Szymanski, H. (2001). Resiliência: Noção, conceitos afins e considerações críticas. In J. Tavares (Ed.), Resiliência e educação (pp. 13-42). São Paulo, SP: Cortez.

Woodhead, M. (2004). Psychosocial impacts of child work: A framework for research, monitoring and intervention. The International Journal of Children's Rights, 12, 321-377. 